\title{
Directing iPSC Differentiation into iTenocytes using Combined Scleraxis Overexpression and Cyclic Loading
}

Angela Papalamprou, $\mathrm{PhD}^{1,2,5}$, Victoria $\mathrm{Yu}, \mathrm{BS}^{1,2,5}$, Angel Chen, $\mathrm{BS}^{1,2,5}$, Tina Stefanovic, $\mathrm{BS}^{1,2,5}$,

Giselle Kaneda, BS ${ }^{1,2,5}$, Khosrowdad Salehi, BS ${ }^{1,2,5}$, Chloe Castaneda, $\mathrm{BS}^{1,2,5}$, Arkadiusz Gertych, $\mathrm{PhD}^{4,6}$, Juliane D Glaeser, $\mathrm{PhD}^{1,2,3,4,5}$, Dmitriy Sheyn, $\mathrm{PhD}^{1,2,3,4,5, *}$

${ }^{1}$ Orthopaedic Stem Cell Research Laboratory, Cedars-Sinai Medical Center, Los Angeles, CA.

${ }^{2}$ Board of Governors Regenerative Medicine Institute, Cedars-Sinai Medical Center, Los Angeles, CA.

${ }^{3}$ Department of Orthopedics, Cedars-Sinai Medical Center, Los Angeles, CA.

${ }^{4}$ Department of Surgery, Cedars-Sinai Medical Center, Los Angeles, CA.

${ }^{5}$ Department of Biomedical Sciences, Cedars-Sinai Medical Center, Los Angeles, CA.

${ }^{6}$ Department of Pathology and Laboratory Medicine

* Correspondence:

Dmitriy Sheyn, PhD

Assistant Professor

Board of Governors Regenerative Medicine Institute

Department of Orthopedics

Department of Surgery

Department of Biomedical Sciences

Cedars-Sinai Medical Center

8700 Beverly Blvd. AHSP A8308

Los Angeles, CA, 90048, USA

Office: (+1)424-315-4579, Fax: (+1)310-594-8924

Email: Dmitriy.Sheyn@csmc.edu 


\begin{abstract}
Regenerative therapies for tendon are falling behind other tissues due to the lack of an appropriate and potent cell therapeutic candidate. This study aimed to induce cell tenogenesis using stable Scleraxis (Scx) overexpression in combination with uniaxial mechanical stretch of mesenchymal stromal cells (MSCs) of different origins. Scleraxis (Scx) is the single direct molecular regulator of tendon differentiation known so far. Mechanoregulation is known to be a central element guiding tendon development and healing. Cells explored were bone marrowderived (BM-)MSCs as well as MSCs differentiated from induced pluripotent stem cells (iMSCs). Upregulation of early and late tendon markers, increased collagen deposition, and morphometric and cytoskeleton-related changes in mechanically stimulated Scx-overexpressing iMSCs compared to BM-MSCs and controls. Our findings suggest that these cells can be differentiated into tenocytes and may be a better candidate for tendon cell therapy applications than BM-MSCs.
\end{abstract}




\section{Introduction}

Tendon and ligament injuries are the main reason for all musculoskeletal consultations worldwide and represent a significant concern in sports medicine and for the general population. An estimated 30 million tendon and ligament cases are reported annually worldwide. ${ }^{1}$ In the US alone, more than 100,000 tendon and ligament repair surgeries are performed annually, resulting in more than $\$ 30$ billion expenditure. ${ }^{2}$ Tendon injuries are associated with a high morbidity, prolonged disabilities, and painful rehabilitation periods, while secondary tendon ruptures often ensue. Current treatment modalities include autografts, allografts, xenografts, suture techniques, and prostheses. $^{2,3}$ These options have major disadvantages including donor site morbidity, risk of limited long-term function, and an incidence of osteoarthritis in up to $50 \%$ of young patients. ${ }^{2,4}$ Following treatment, functional recovery of repaired tendons is usually incomplete. The mechanical and structural properties of repaired tissue are permanently altered and fail to reach the level of functionality achieved prior to injury. ${ }^{5}$ Development of a cell therapy approach, which could be applied to an injured tendon or ligament site, could dramatically alter patient outcomes.

Adult stem/progenitor cells that have been identified in the tendon niche and characterized by their marker profile are considered promising cell sources for tendon tissue engineering. ${ }^{6}$ However, they are very scarce in vivo and cannot be expanded in vitro for therapeutic applications due to phenotypic drift in culture. ${ }^{7}$ Thus, adult stem/progenitor cell-mediated therapies have shown limited potential to be used for tendon repair. ${ }^{8-10}$ In addition, mesenchymal stromal cells (MSCs) derived from bone marrow (BM-MSCs) or adipose tissue (ASCs) have been explored as a potential tendon repair and tissue engineering strategy due to their abundance, multipotency, and regenerative potential in vivo. Several animal models have shown improved 
functional outcomes and enhanced tendon healing via utilizing MSCs. ${ }^{8,9,11}$ However, their major disadvantages are their limited self-renewal capacity, phenotypic heterogeneity, and potential for ectopic bone or cartilage formation. These attributes may limit their clinical application due to the need for in vitro expansion in adequate numbers and thorough characterization to meet regulatory standards prior to clinical use. ${ }^{5}$ Additionally, their tenogenic potential has shown to be restricted. ${ }^{12}$ Developing an off-the-shelf cell source that can be efficiently differentiated to tenogenic progenitor cells is a prerequisite for tendon cell-based therapy success.

The discovery of induced pluripotent stem cells (iPSCs) through nuclear reprogramming of somatic cells revolutionized the field of regenerative medicine, because of their high selfrenewal capacity and unparalleled developmental plasticity. Additionally, iPSCs might have the advantage of being able to evade the recipient's immune response. ${ }^{13,14}$ iPSCs have been successfully differentiated to MSC-like cells (iMSCs) by our group and others. ${ }^{15,16}$ One of the main advantages to the use of iMSCs is that they potentially represent an unlimited source for tenocytes. Small animal tendon repair models utilizing iPSCs have shown improved functional outcomes. $^{17,18}$ Thus, iMSCs derived from iPSCs may offer an unlimited off-the-shelf allogeneic source for tendon cell therapy applications.

Development of tenocytes occurs in at least two stages: First, tenocyte progenitors (tenoblasts) express scleraxis $(\mathrm{Scx})$, which is the single direct molecular regulator of tendon differentiation known to date. ${ }^{19,20}$ Second, tenocyte maturation results in tissue formation. ${ }^{20}$ However, ectopic overexpression of Scx in BM-MSCs was not found sufficient to drive tenogenesis. ${ }^{19,21}$ Tendon and other tissues grow and remodel in response to changes in their environment. At the cell level, spatial distribution of dynamic mechanical cues can affect developmental, maintenance and healing responses. $^{22,23}$ Mechanoregulation has been shown to be a central element guiding 
embryonic tendon development and healing. ${ }^{24}$ Fully differentiated cells, such as tenocytes and fibroblasts, can actively sense both the external loading applied to them and the stiffness of their environment. ${ }^{24,}{ }^{25}$ Mechanical cues can also affect the differentiation of multipotent cells such as BM-MSCs. ${ }^{26,27}$ Additionally, tenocytes and tendon progenitors are able to retain their phenotype when they are stretched or cultured on mechanovariant substrates with controlled mechanical properties. ${ }^{28}$ Uniaxial cyclic stretching has been used to drive tenogenic differentiation in vitro, as it is considered more relevant physiologically. ${ }^{29}$ Therefore, mechanical stimulation may be essential for the differentiation of multipotent cells towards tenogenic progenitors, as well as for the maturation of the tenocyte phenotype.

Tenocytes can actively sense external loading, retain their phenotype when they are stretched, and they dedifferentiate when cultured statically. Additionally, Scx is a crucial factor for tenogenic differentiation. ${ }^{7,19,20}$ Therefore, combinatory mechanical and biological stimulation may be essential for tenogenesis. We hypothesized that iMSCs can be efficiently differentiated into tenocytes by combination of stable overexpression of Scx and mechanical stimulation in vitro. In this study we investigated (a) the ability of Scx stable overexpression to induce tenogenic differentiation, and (b) the effect of mechanical stimulation to guide tenogenic differentiation in vitro with and without Scx overexpression. To accomplish this, Scx was overexpressed using lentiviral vectors. BM-MSCs, BM-MSC ${ }^{\mathrm{SCX}+}$, iMSCs and $\mathrm{iMSC}^{\mathrm{SCX}+}$ cells were grown on mechanovariant substrates. Cyclic uniaxial stretching was applied on iMSCs and iMSC $^{\mathrm{SCX}+}$ using the CellScale MCFX bioreactor system. The effect of Scx overexpression with and without mechanical stimulation was assessed with gene expression analyses and immunocytochemistry for tendon markers, collagen deposition, and morphometric analysis of cytoskeletal orientation following uniaxial stretching. 


\section{Methods}

\section{Primary cell isolation and expansion}

Human bone marrow was acquired from Lonza (Allendale, NJ) and BM-MSCs were isolated as previously reported..$^{30-32}$ Briefly, bone marrow was overlayed on Ficoll-paque density gradient medium (GE healthcare, Chicago, IL), washed twice with phosphate buffered saline (PBS), plated at a density of $2 \times 10^{5} / \mathrm{cm}^{2}$ (Corning, Corning, NY), and incubated overnight at $37^{\circ} \mathrm{C} / 5 \%$ $\mathrm{CO}_{2}$. After $48 \mathrm{~h}$, non-adherent cells were discarded, washed with phosphate buffered saline (PBS), and culture medium was added, composed of $2 \mathrm{mM}$ L-glutamine, $1 \%$ antibiotic antimycotic solution (AAS) in Dulbecco's modified eagle medium (DMEM, all from Thermofisher, Waltham, MA), and 20\% fetal bovine serum (FBS, GeminiBio, West Sacramento, CA). BM-MSCs were isolated via adherence and cultured at $37^{\circ} \mathrm{C} / 5 \% \mathrm{CO}_{2}$. $\mathrm{M}$ edia were switched to standard MSC culture media (low glucose DMEM, 1\% AAS, 10\% FBS, 2mM L-glutamine) and were changed every 3 days. Cells were split upon confluence in a 1:3 ratio.

Primary porcine tenocytes were isolated from pig Achilles tendons. Briefly, tendons were washed with PBS containing antibiotics, manually minced to 2-3mm fragments, and digested with $0.02 \%$ collagenase II (LS004205, Worthington Biochemical Corporation, Lakewood, NJ) for $18 \mathrm{~h}$ at $37^{\circ} \mathrm{C} / 5 \% \mathrm{CO}_{2}$ with rocking. The next day, pre-warmed media were added (high glucose DMEM supplemented with 1\% AAS, 10\% FBS and 2mM L-glutamine), and tissue digests were centrifuged and strained through a $70 \mu \mathrm{m}$ filter. Isolated cells were cultured in high glucose DMEM supplemented with 1\% AAS, 10\% FBS and 2mM L-glutamine. 


\section{iMSC derivation and expansion}

Normal human iPSC lines were obtained from the Cedars-Sinai iPSC core facility, expanded on Matrigel $^{\mathrm{TM}}$-coated plates (Corning) with chemically defined mTeSR ${ }^{\mathrm{TM}}$ Plus media (StemCell Technologies, Vancouver, Canada). iMSCs were differentiated from human iPSCs and expanded in vitro using our previously published method. ${ }^{15}$ iMSCs were maintained following the same protocol and media as described for BM-MSCs. Media were changed every 3 days, and cells were split upon $80 \%$ confluence in 1:3 ratio.

\section{Genetic engineering of BM-MSCs and iMSCs to overexpress Scx}

BM-MSCs and iMSCs were engineered via lentiviral transduction to overexpress Scx-GFP under the constitutively active CMV promoter coupled to its enhancer using a lentiviral vector (BM$\left.\mathrm{MSC}^{\mathrm{Scx}-\mathrm{GFP}+}, \mathrm{iMSC}^{\mathrm{Scx}-\mathrm{GFP}+}\right){ }^{33,34}$ Briefly, HEK293T/17 cells (ATCC) were seeded at the density of $6 \times 10^{4} / \mathrm{cm}^{2}$ in Eagle's Minimum Essential Medium (EMEM, Thermofisher, Waltham, MA), containing $10 \% \mathrm{FBS}$, and 1\% AAS. Lentiviruses were produced by transfecting HEK293T/17 cells with the pLenti-C-mGFP vector where SCXB has been inserted (OriGene, Rockville, MD) expressing Scx-GFP and two packaging plasmids ${ }^{33}$ (pCMV-dR8.2, pCMV-VSV-G). Transfections were carried out using the BioT method with a 1.5:1 ratio of BioT ( $\mu$ l) to DNA $(\mu \mathrm{g})$. The virus-containing medium was harvested at 48 and $72 \mathrm{~h}$ after transfection. Both batches were combined, centrifuged, filtered, and used to transduce BM-MSCs and iMSCs within 24h. Transduction titers were determined using flow cytometry for GFP and verified using RT-qPCR for Scx. 


\section{Flow cytometry}

For assessment of lentiviral transduction efficiency, transduced cells were washed with PBS and digested with $0.05 \%$ Trypsin-EDTA (Gibco) for $5 \mathrm{~min}$ at $37^{\circ} \mathrm{C}$. Cells were then washed with FACS buffer containing 2\% bovine serum albumin (BSA, A4503, Sigma, St. Louis, MO) and 0.1\% sodium azide (S2002, Sigma) in 1x PBS and were acquired on a BD LSR Fortessa analyzer (BD Bioscences, San Jose, CA). Generated FSC files were analyzed using Flowjo software (Flowjo LLC, Ashland, OR).

\section{Cell culture on various stiffness surfaces}

BM-MSCs and iMSCs were seeded at $2.5 \times 10^{4} / \mathrm{cm}^{2}$ in plates of varying stiffness. Specifically, cells were seeded into 6-well Cytosoft ${ }^{\circledR}$ (Advanced Biomatrix, Carlsbad, CA) plates which are pre-coated with polydimethylsiloxane (PDMS) by the manufacturer with (E) of E 2kPa and $\mathrm{E} \sim 32 \mathrm{kPa}$, as well standard tissue culture polystyrene (TCP) plates (E GPa range) (Corning). Prior to seeding the cells, PDMS plates were coated with Collagen-1 (Purecol ${ }^{\circledR}$, Advanced Biomatrix), following the manufacturer's recommendations. Cells were grown for two weeks in tenogenic media (low glucose DMEM supplemented with $2 \mathrm{mM}$ glutamine, $10 \%$ FBS, $1 \%$ AAS, and $50 \mu \mathrm{g} / \mathrm{ml}$ ascorbic acid). ${ }^{35,36}$ After 2 weeks, cells were lysed with RLT plus lysis buffer (RLT plus buffer, Qiagen, Valencia, CA containing $\beta$-mercaptoethanol, M3148, Sigma, St. Louis, $\mathrm{MO})$, and stored at $-80^{\circ} \mathrm{C}$ until processing for gene expression analysis.

\section{Mechanical loading}


Since tendon cells predominantly receive uniaxial tensile loading from collagen bundles in vivo, ${ }^{29}$ the CellScale MCFX bioreactor (CellScale Biomaterials Testing, Waterloo, ON, Canada) was chosen, as it allows for longitudinal stretching on the cell monolayer. Cells were seeded at a density of $2 \times 10^{4} / \mathrm{cm}^{2}$ on fibronectin-coated silicone plates (CellScale Biomaterials Testing) and allowed to attach for $24 \mathrm{~h}$. Since the strain gradients in developing tissues are not well-known, the cyclic stretching protocol was optimized based on published literature at $4 \%$ sinusoidal strain, $0.5 \mathrm{~Hz}, 2 \mathrm{~h} /$ day. $^{37,38}$ Cells seeded on the same fibronectin-coated silicone plates were placed in the incubator in parallel and served as static controls for those experiments. iMSCs and iMSC ${ }^{\mathrm{Scx}+}$ were stretched for up to 7 days, and media were changed 3x/week in tenogenic media (also used for in vitro static culture). Cells were harvested on days 0,3 and 7 ( $n=8 /$ timepoint). In the case of iMSCs at 7d, two adjacent wells were pooled to make one lysate $(n=4$, representing 8 total wells).

\section{Cell morphology and morphometric analysis}

At all time-points (day 3 and 7), cells were fixed with 2\% paraformaldehyde for 15 min at RT, permeabilized in PBS with 0.2\% Triton X-100 (PBS-T), and stained with anti-F-actin iFluor 555 phalloidin antibody (Abcam, Cambridge, UK) to identify the cytoskeleton. Nuclei were counterstained with DAPI (Thermofisher). Samples were imaged in the inverted Revolve fluorescent microscope (Revolve, Echo, San Diego, CA) with a 200x magnification objective using a 3x3 grid to capture 9 non-overlapping images for analysis. Morphometric analysis of cells was carried out using a custom analytical pipeline developed in MATLAB (Matlab Natic, MA), as previously described. ${ }^{39}$ This analysis was performed in unprocessed single channels by a bioinformatician, who was given access to the files and was blinded to the groups. Briefly, size 
and shape of nuclei in the nuclear mask was outputted by an algorithm, which processed the DAPI image characterized by orientation, area, and aspect ratio features as previously described. ${ }^{39}$ Nuclei were used to determine cell density and were also analyzed for their orientation and aspect ratio (width versus length of the nucleus). To quantify organization of cytoskeleton in each cell, ImageJ directionality tool was used to assess actin filament angle distributions.

\section{Gene expression analysis}

Differentiation to iTenocytes (iTCs) was defined based on expression of tendon-specific markers (Suppl. Table 1) using RT-qPCR with TaqMan ${ }^{\circledR}$ gene expression as well as osteogenic and adhesion markers. ${ }^{40,41}$ Total RNA was isolated to analyze the gene expression of tendon phenotypic markers (Suppl. Table 1). For RNA isolation, media were aspirated, and cells lysed with RLT plus lysis buffer (Qiagen). Lysates were transferred to $1.5 \mathrm{ml}$ tubes and were fully homogenized using a handheld pestle and mortar. RNA was isolated using the RNeasy plus mini kit (Qiagen) following the manufacturer's recommendations. RNA yields were determined spectrophotometrically using a Nanodrop system (Thermofisher), and RNA was reversetranscribed with the high-capacity cDNA reverse transcription kit (Applied Biosystems). Target gene mRNA levels were quantified using FAM-MBG technology (Bio-Rad, Hercules, CA). The threshold cycle $(\mathrm{Ct})$ value of $18 \mathrm{~S}$ rRNA was used as an internal control using the TaqMan ${ }^{\circledR}$ gene expression FAM/MGB probe system (4333760F, Thermofisher). The Livak method was used to calculate $\Delta \Delta \mathrm{Ct}$ values and fold change was calculated as $2^{-\Delta \Delta \mathrm{Ct}}$, as previously described. ${ }^{42}$ 


\section{Assessment of collagen deposition}

Newly synthesized collagen was quantified using the Sirius red total collagen assay detection kit following the manufacturer's protocol (Chondrex, Redmond, WA). Total collagen in each cell culture media sample was concentrated using Concentrating Solution (Chondrex) at $4^{\circ} \mathrm{C}$ for 18 24h. Following incubation, samples were centrifuged for $3 \mathrm{~min}$ at $10,000 \mathrm{rpm}$. Briefly, collagen pellets were dissolved in $0.05 \mathrm{M}$ acetic acid (Sigma), precipitated with Sirius Red dye, washed, and then transferred to a 96-well plate. Optical density at $535 \mathrm{~nm}$ was determined for samples and assay standards. Collagen concentration was calculated from the standard curve using regression analysis and plotted in $\mu \mathrm{g} / \mathrm{ml}$ units. All experimental samples $(\mathrm{n}=8)$ and assay standards were conducted in technical triplicates.

\section{Immunocytochemistry (ICC)}

Cell cultures of $\mathrm{iMSC}^{\mathrm{SCX}+}$ were grown in static culture or uniaxially stretched in the $2 \mathrm{D}$ bioreactor for 7 days as described above. After 7 days, cells were briefly washed with PBS and fixed with $4 \%$ paraformaldehyde for $15 \mathrm{~min}$ at RT. Cells were washed again, and nonspecific sites were blocked with 4\% normal donkey serum (Jackson Immunoresearch, West Grove, PA) in $0.1 \% \mathrm{v} / \mathrm{v}$ PBS-Tween ${ }^{\circledR} 20$ Detergent $(\mathrm{PBS}-\mathrm{T})$ for $45 \mathrm{~min}$. After, cells were incubated at $4^{\circ} \mathrm{C}$ overnight with the primary antibody for Tenomodulin (HPA055634, Sigma, Darmstadt, Germany). Cells were then washed 3 times with PBS-T, followed by a $2 \mathrm{~h}$ incubation at RT with the secondary antibody donkey anti-rabbit Alexa Fluor ${ }^{\circledR}$ 647-conjugated Affinipure (Jackson Immunoresearch). Nuclei were counterstained with DAPI. Fluorescent images were captured with a Revolve fluorescent microscope (Revolve). Images of single channels were taken, and they were merged either during imaging or using Adobe Photoshop CS6 post imaging. Adobe 
Illustrator CS6 was used for final figure production. BioRender software program was used to prepare the graphical abstract and additional graphics used throughout the manuscript.

\section{Statistical Analysis}

All data are presented as mean \pm standard deviation from the mean. Normally distributed data were analyzed with unpaired t-test (for 2 groups) or non-repeated measures analysis of variance followed by Tukey-Kramer HSD post hoc analysis when more than 2 groups were compared. Non-parametric data were analyzed using the Friedman test. To compare gene expression levels between pig tenocytes cultured on different plates over time, a mixed repeated measures model was used, followed by Tukey's post hoc tests for between group comparisons. Lastly, to analyze the orientation of nuclei and actin filaments between the static and stretched conditions, medians were compared using the Mann-Whitney test, while the two frequency distributions were compared using the Kolmogorov-Smirnov test. Statistical significance was set at $\mathrm{p}<0.05$. Statistical analyses were performed with GraphPad Prism 9.0.

\section{Results}

\subsection{Confirmation of stable lentiviral-driven Scx overexpression in ${\mathrm{BM}-M S C^{S c x+}}^{\text {and }} \mathrm{iMSC}^{\mathrm{SCX}+}$}

We generated lentiviral vectors expressing Scx tagged with GFP at the C-terminus and under the constitutively active CMV promoter, as previously described. ${ }^{33,34}$ The absolute intensity of GFPpositive cells was used as a proxy for Scx integrations using flow cytometry. Absolute GFP fluorescence was similar for the highest titers (Suppl. Fig. 1A). A dose-response effect of lentiviral load in transduction efficiency was observed when flow results were presented as percentage of GFP+ cells (Supp Fig. 1B). We expanded BM-MSCs and iMSCs transduced with 
Scx-GFP lentivirus vector (BM-MSC ${ }^{\mathrm{SCX}+}$ and $\mathrm{iMSC}^{\mathrm{SCX}+}$ ) and assessed the expression of Scx at 4 weeks of regular culture without sorting. Scx expression was significantly upregulated in BM$\mathrm{MSC}^{\mathrm{SCX}+}$ and $\mathrm{iMSC}^{\mathrm{SCX}+}$ showing stable overexpression of Scx at after 4 week 4 (Suppl. Fig. 1C).

\subsection{Tenogenic markers gene expression of BM-MSCs, BM-MSC ${ }^{S C X+}$, iMSCs and iMSC ${ }^{S C X+}$}

\section{after 2 weeks of static culture}

First, we examined the effect of Scx overexpression on BM-MSCs and iMSCs in vitro. The four cell groups BM-MSCs, BM-MSC ${ }^{\mathrm{SCX}+}$, iMSCs, and $\mathrm{iMSC}^{\mathrm{SCX}+}$ were generated and cultured in vitro on TCP for a duration of 2 weeks (Fig 1A). Scx was demonstrated to be significantly overexpressed in $\mathrm{BM}-\mathrm{MSC}^{\mathrm{SCX}+}$ and $\mathrm{iMSC}^{\mathrm{SCX}+}$ compared to BM-MSCs and iMSCs after 2 weeks (Fig 1B). Findings on TCP for 2 weeks were summarized in a heat map showing changes compared to baseline (Fig.1C) and as bar graphs displaying differences between all cell types (Fig. 1D). Tenogenic marker genes (Mkx, Thbs4, and Tnmd) and ECM proteins previously associated with tenogenesis (Bgn, Col3a1, and Dcn) were significantly upregulated in iMSC ${ }^{\mathrm{SCX}+}$ compared to their own baseline (day 0) whereas in BM-MSC ${ }^{\mathrm{SCX}+}$ only Bgn, Thbs4, and Den were significantly upregulated compared to their own baseline (Fig1C). Mkx and Tnc showed significant downregulation in $\mathrm{BM}-\mathrm{MSC}^{\mathrm{SCX}+}$ and in BM-MSCs. When compared to all other cell types, in the $\mathrm{iMSC}^{\mathrm{SCX}+}$ group, expression of Mkx, Bgn, Thbs4, and Tnmd was significantly higher. Thbs4 was significantly higher in $\mathrm{BM}-\mathrm{MSC}^{\mathrm{SCX}+}$ versus BM-MSCs. However, Tnmd was

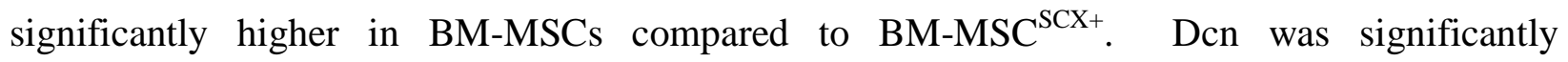
upregulated in $\mathrm{iMSC}^{\mathrm{SCX}+}$ but was lower compared to both BM-MSCs and BM-MSC ${ }^{\mathrm{SCX}+}$ (Fig. 1D). 
bioRxiv preprint doi: https://doi.org/10.1101/2021.11.23.469329; this version posted November 23, 2021. The copyright holder for this preprint (which was not certified by peer review) is the author/funder, who has granted bioRxiv a license to display the preprint in perpetuity. It is made available under aCC-BY 4.0 International license. 


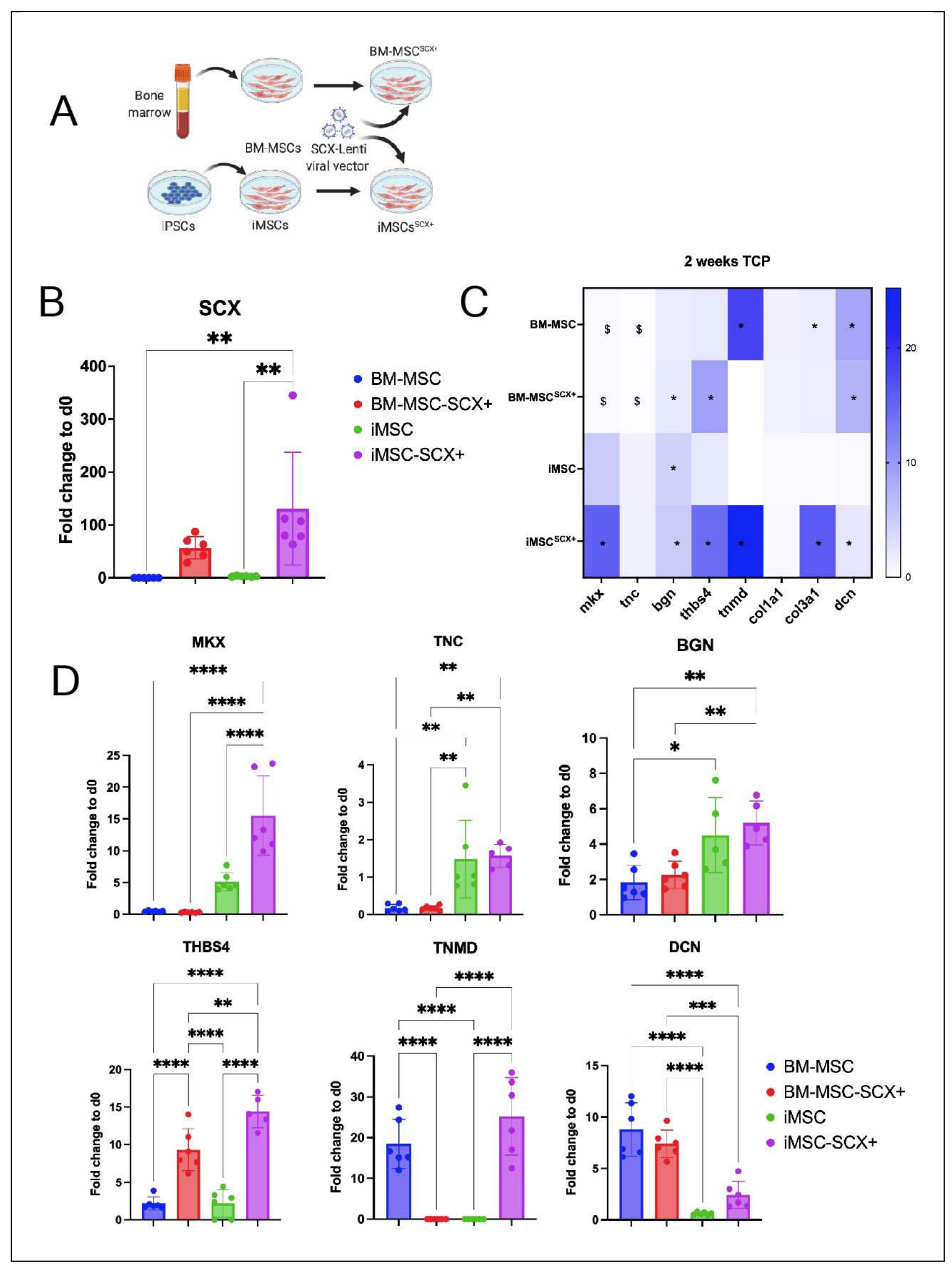




\section{Fig. 1. iMSC $^{\text {Scx+ }}$ show higher tenogenic potential than other cell types. (A). Graphical} representation of the generation of four cell types that were used in the static culture differentiation experiments. BM-MSCs and iMSCs were transduced with lentiviral vectors to overexpress Scx stably. Cells (BM-MSCS, BM-MSC $C^{S C X+}$, iMSCs and iMSC $C^{S C X+}$ ) were maintained in vitro for 2 weeks and then gene expression analyses were performed. (B) Gene expression analysis for Scx between the four groups. $n=6$ replicates/group. $(C)$ Tenogenic marker expression levels are displayed as heat maps; $n=6, *_{p}<0.05$, (significant upregulation compared to baseline); ${ }^{\$} p<0.05$ (significant downregulation compared to baseline). (D) Gene expression levels of tenogenic markers were different between the four cell types after 2 weeks of culture in vitro. Between group comparisons were performed using one-way ANOVA with Tukey's post- hoc; $n=6, *_{p}<0.05$, **p<0.01, ***p<0.001, ****p<0.0001.

\subsection{Static culture of $B M-M S C s, B M-M S C^{S C X+}$, iMSCs, and iMSC ${ }^{S C X+}$ under differential substrate stiffness conditions}

First, we examined the effect of differential substrate stiffness $(\mathrm{E} \sim 2 \mathrm{kPa}, 32 \mathrm{kPa}$ versus regular TCP) on tenocyte marker expression (Col1a1, Col3a1, Tnmd, Thbs4,Mkx and Alpl) of primary porcine tenocytes for a duration of 8 cell passages (Suppl. Fig. 2). Col1a1 gene expression was detected until passage 8 in porcine tenocytes cultured on all types of surfaces, and there was a significant increase in Colla1 in the early passages on all 3 substrates compared to baseline. Although Tnmd was slightly downregulated with passaging, it remained close to baseline in all three substrates until P7 when it dropped to 0 for $\mathrm{E} \sim 2 \mathrm{kPa}$ and $\mathrm{TCP}$, while it was still close to baseline for E 32kPa. However, by P8 its expression had dropped to 0 in all substrates. In the case of Thbs 4 baseline expression was retained until P5 for the two softer substrates and then dropped to 0 , while on TCP it was retained until P7. Alpl was significantly upregulated in TCP compared to both softer substrates and compared to baseline. Mkx was significantly 
downregulated after 1 passage in all substrates and remained at the same lower levels compared to baseline expression (at day 0) until the end of the study (data not shown). Lastly, Col3a1 expression was doubled compared to baseline in all substrates and remained constant until the end of the study. Next, the effect of substrate stiffness on the BM-MSC and BM-MSC ${ }^{\text {SCX+ }}$ tenogenic differentiation potential was investigated. Tenogenic marker expression was increased in the TCP group. Scx was significantly higher in $\mathrm{BM}-\mathrm{MSC}^{\mathrm{SCX}+}$ cultured on TCP versus all other groups. Furthermore, Col1a1, Mkx, Thbs4, Bgn, and Den were significantly higher in BM$\mathrm{MSC}^{\mathrm{SCX}+}$ cultured on TCP compared to the two softer substrates (Supp. Fig. 3). Lastly, the effect of differential substrate stiffness on iMSC and $\mathrm{iMSC}^{\mathrm{SCX}+}$ differentiation was assessed (Fig. 2A). Tnc and Bgn were significantly higher in iMSCs and iMSC ${ }^{\mathrm{SCX}+}$ cultured on TCP compared to the lower stiffness substrates in iMSCs (Fig. 2B). Tenogenic markers Scx, Mkx, Tnmd, and Thbs4 were significantly upregulated in $\mathrm{iMSC}^{\mathrm{SCX}+}$ cultured on TCP compared to iMSCs and iMSC ${ }^{\mathrm{SCX}+}$ cultured in all other substrates. Col1a1 and Col3a1 were close to baseline expression levels in iMSC $^{\mathrm{SCX}+}$ cultured on TCP (data not shown). Zero net secretion of collagen was found in BMMSCs, BM-MSC ${ }^{\mathrm{SCX}+}$, iMSCs and $\mathrm{iMSC}^{\mathrm{SCX}+}$ compared to baseline levels on the three different substrates after 2 weeks (data not shown). 


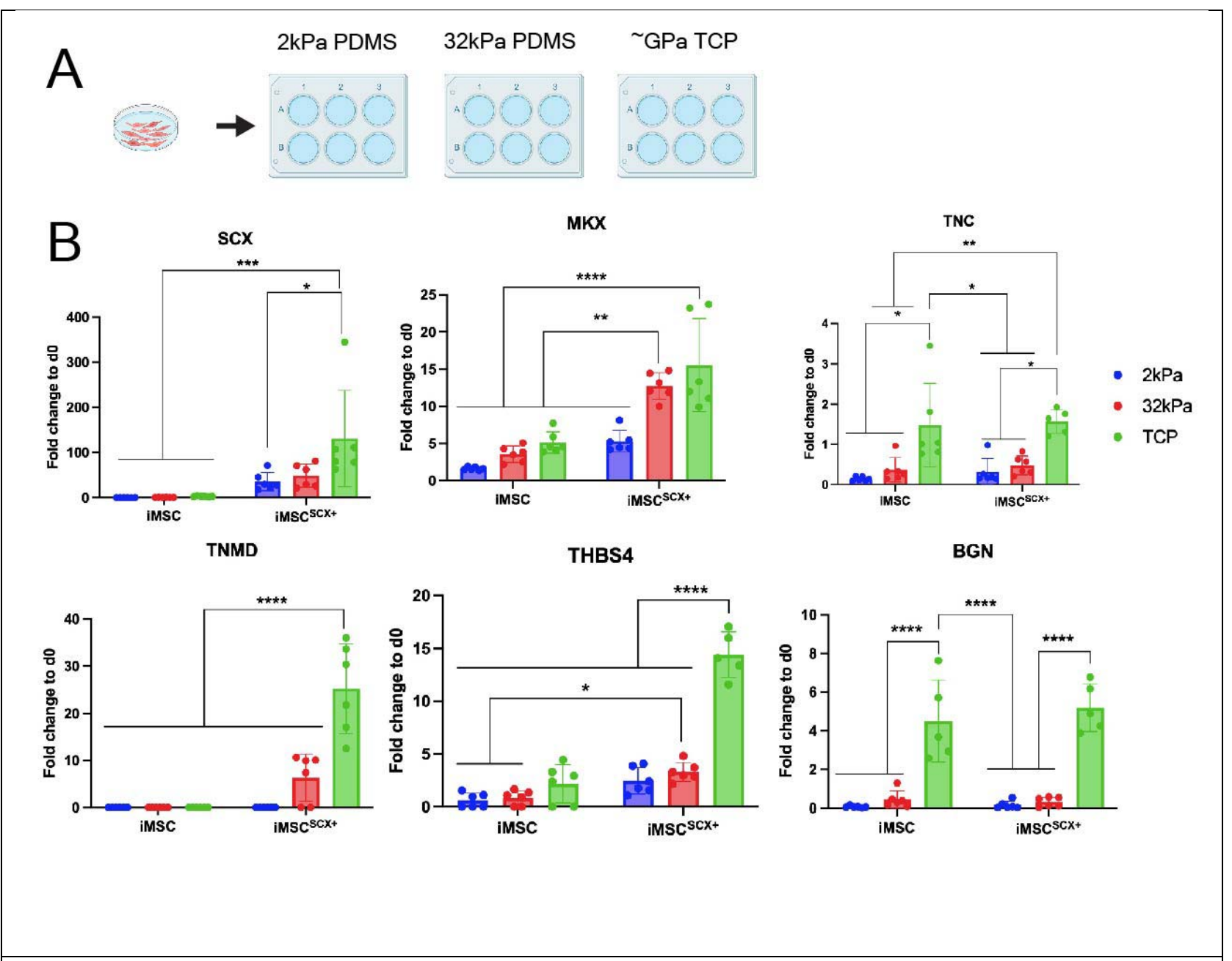

Fig 2. Lower substrate stiffnesses do not promote tenogenic differentiation in iMSCs with and without Scx. (A) Cells (iMSCs and iMSC $C^{S C X+}$ ) were differentiated in vitro for 2 weeks on surfaces with different substrate stiffnesses $(2 \mathrm{kPa}, 32 \mathrm{kPa}$, and TCP that is $\sim \mathrm{GPa})$. (B) Gene expression analyses were performed. $n=6$ replicates/group. ${ }^{*} p<0.05, * * p<0.01, * * * p<0.001, * * * * p<0.0001$

\subsection{Dynamic culture of iMSCs and iMSC ${ }^{S C X+}$ using cyclic uniaxial stretching}

Tenogenic marker expression of iMSCs cultured on silicon plates in static conditions or cyclic stretching was analyzed at 3 and 7 days (Fig. 3A). At 7 days, Scx was upregulated compared to day 3 and the static group. At day 3, Scx, Mkx, Col3a1, and Pdgfra had an upward trend. At day 7, Col1a1, Tppp3, Pdgfra, and Tnmd were significantly upregulated compared to the static 
group. Thbs4 was upregulated compared to day 3 and to baseline, but it was not significantly altered between the static and stretched groups in iMSCs.

Cyclic stretching of $\mathrm{iMSC}^{\mathrm{SCX}+}$ for 3 days resulted in an upregulation of Mkx, Thbs4, Bgn, Col1a1, and Col3a1. At day 7, Thbs4 was still upregulated, Sox9 was also significantly upregulated, while Mkx dropped to baseline levels (Fig. 3B). Colla1 and Col3a1 were significantly upregulated after 3 and 7 days of stretching. Interestingly, in iMSC ${ }^{\mathrm{SCX}+}$ baseline expression of Col1a1 and Col3a1 was significantly downregulated compared to iMSC baseline expression (Suppl. Fig. 4). Newly synthesized secreted collagen was significantly higher after 7 days of cyclic stretching in $\mathrm{iMSC}^{\mathrm{SCX}+}$ that were stretched compared to iMSCs that were also stretched for the same time, as well as both cell types that were cultured for 7 days in the silicone plates (static conditions, Fig 3C). 


\section{A $\underset{\text { imsos }}{-1}$}
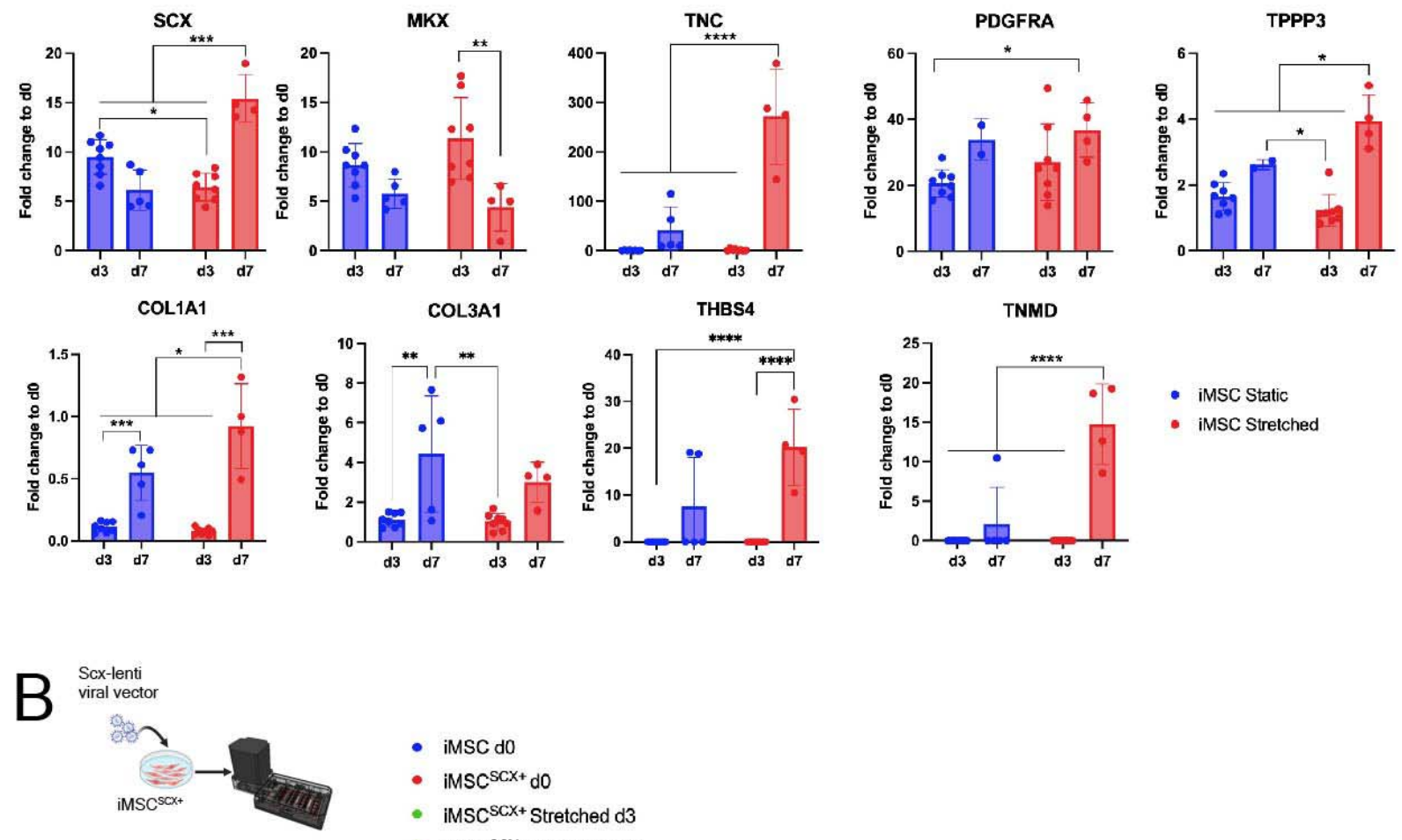

- iMSC d0

- iMSC ${ }^{S C X}+$ Stretched d3

- iMSC $5 \mathrm{CX}+$ Stretched d7
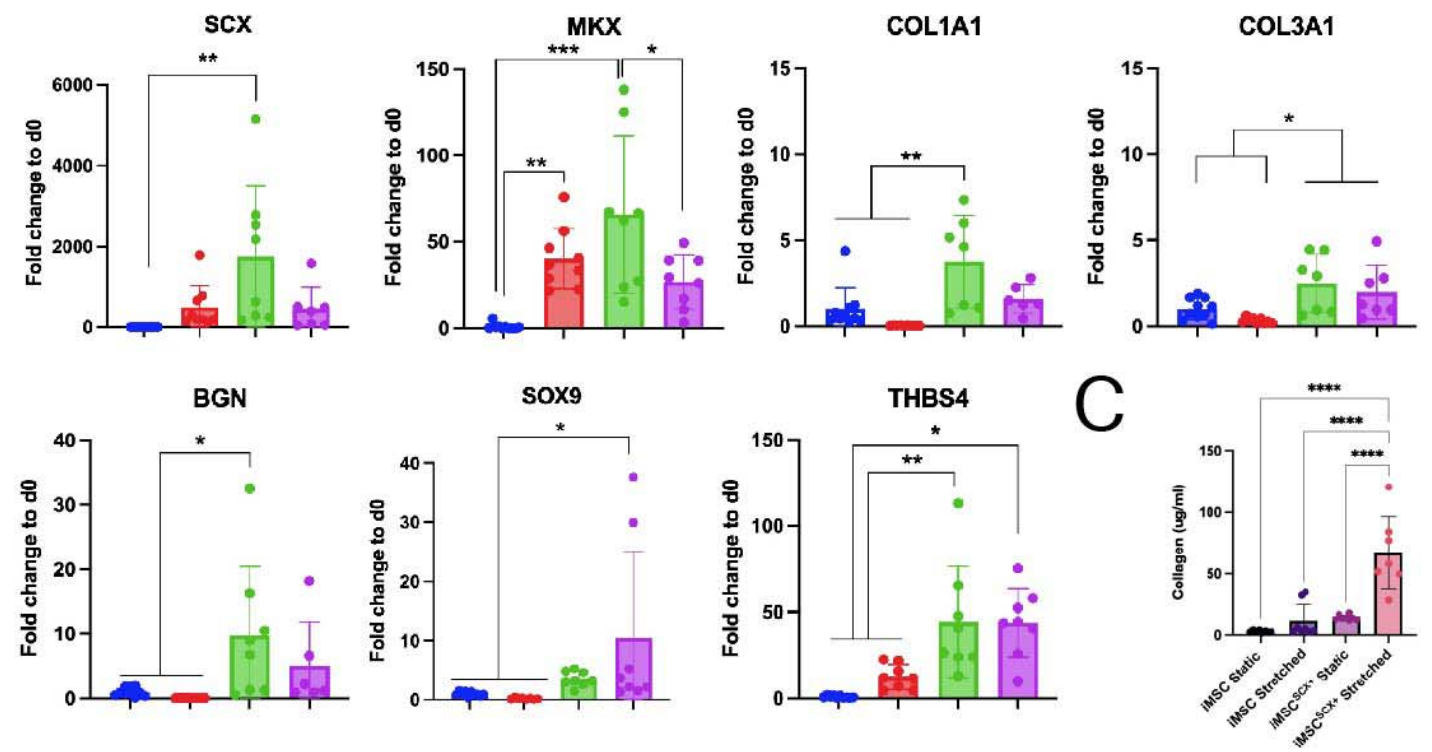
Fig. 3. Tenogenic markers gene expression and matrix secretion are stimulated by Scx overexpression and uniaxial stretch in $2 D$ bioreactor. iMSCs and $i M S C^{S C X+}$ were stretched in a $2 D$ bioreactor for 7 days. For static controls, cells were plated in matching plates with no stretching. (A) Gene expression analyses for iMSCs stretched for 3 days and 7 days in the $2 D$ bioreactor. (B) Gene expression analyses for $i M S C^{S C X+}$ stretched for 3 days and 7 days in the $2 D$ bioreactor. $(C)$ Collagen deposition following 7 days of stretch in the 2 D bioreactor. $n=8 /$ group $; *_{p}<0.05$, **p<0.01, ***p<0.001, $* * * * p<0.0001$

Cellular morphology and orientation of $\mathrm{iMSC}^{\mathrm{SCX}+}$ was examined after 7 days of uniaxial loading or static culture via phalloidin staining of the actin fibers of the cytoskeleton. Actin filaments aligned perpendicular to the axis of the load in the stretched plates, whereas a stochastic cytoskeleton alignment was observed in the static culture (Fig. 4A). Additionally, an unbiased morphometric analysis was conducted on both culture conditions by using high content image analysis of the nuclei, whereby the operator of the software was blinded to the groups. Cell density was significantly higher in the static compared to the stretched culture condition (Fig. 4B). Nucleic aspect ratio medians were similar for both conditions (Fig. 4C). The frequency distribution of the nuclei showed that in both conditions $76 \%$ of all nuclei had aspect ratios between 0.55-0.8 (Fig. 4C). Nucleic orientation assessment, however, showed that uniaxial cyclic loading for 7 days significantly affected the frequency distribution of nuclear orientation, which was assessed as an angle from the axis of loading (depicted in Fig. 4D). The medians of nuclear orientation of the static and the stretched conditions were shown to be significantly different. After 7 days. $42.53 \%$ of stretched nuclei displayed an angle of $65-90^{\circ}$, as opposed to only $26.5 \%$ of the nuclei of the static culture (Fig. 4D). In contrast, $30.19 \%$ of the static culture nuclei displayed an angle of $0-20^{\circ}$, while only $15.1 \%$ in the stretched plate were found in the 
same angular range (Fig. 4D). Furthermore, the frequency distribution of nuclear angles was skewed to the right, as opposed to the static culture, which showed a stochastic alignment of nuclei in all directions. Actin filaments displayed a different orientation distribution in the static culture versus the stretched condition. In the static condition, there were similar fractions of fibers aligned in each directionality cluster. In the stretched condition, $>50 \%$ of fibers were found to be aligned in $80-120^{\circ}$. Even though the two medians were not statistically different, the frequency distributions between the two conditions were shown to be significantly different. 


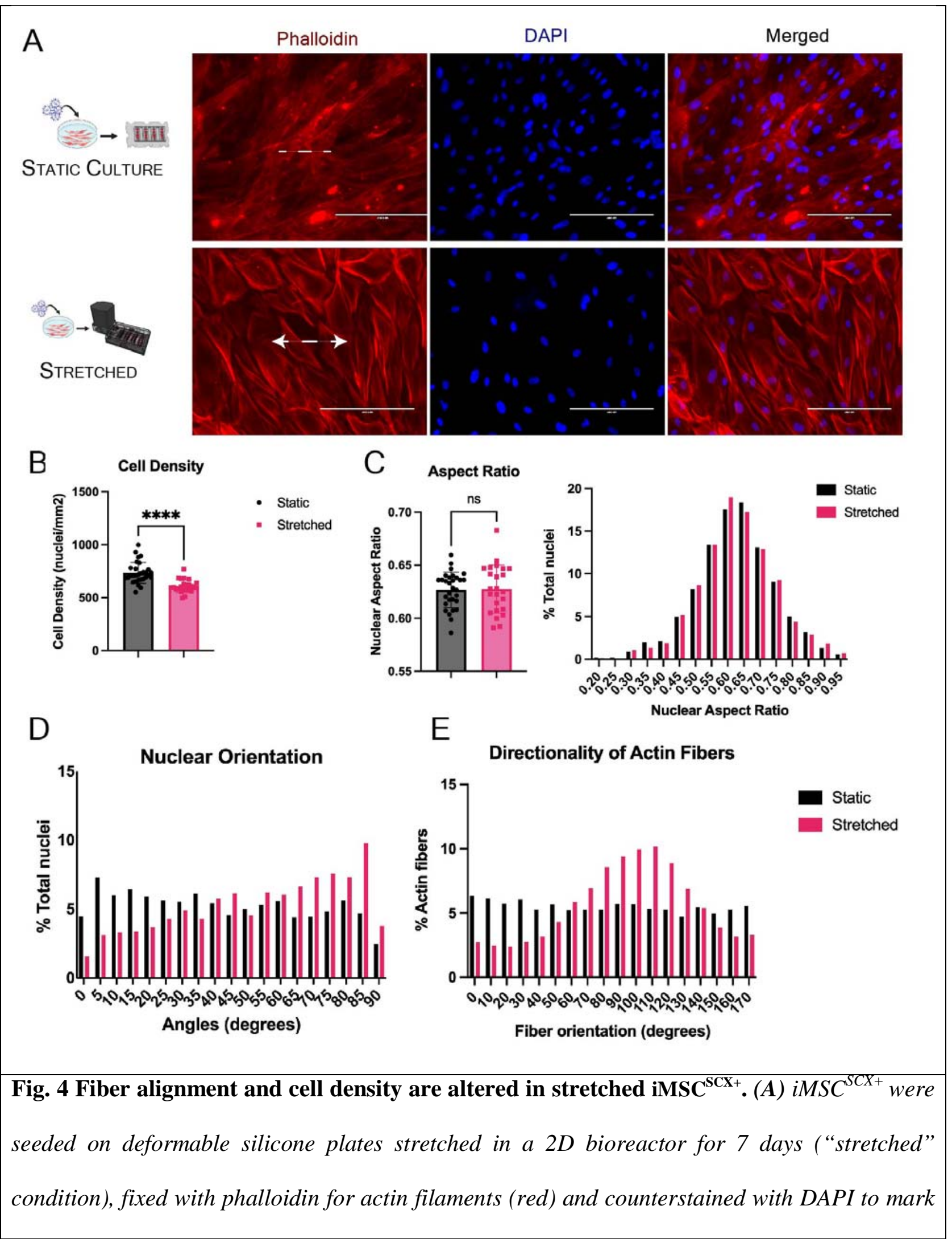


nuclei (blue). Cells were also plated on similar plates that were placed in the incubator for the same period (static culture controls, "static"). White arrows display the direction of applied

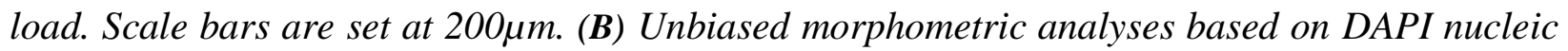
staining were performed to assess the effect of uniaxial cyclic loading on cellular orientation. Cell density was computed from quantifying the nuclei in each well (n=12/group). (C) Aspect ratio (width versus length of the nucleus) was computed to assess whether nuclei were elongated in each state. Values close to 1 reflect rounded nuclei, whereas smaller fractions represented more elongated nuclei. The frequency distribution of all nuclei aspect ratios is also presented as $\%$ percentage of total cells. Both representations of the aspect ratio show no difference in nuclei elongation. (D) The frequency distribution of nuclear orientation angles was plotted for the two conditions. Both the distributions and their individual medians in the two tested conditions were significantly different and the stretched cells displayed skewness to the right. (E) The frequency distribution of actin filament angles was plotted for the two conditions. The two distributions were significantly different from each other and $>50 \%$ of filaments of the stretched condition were found to be in $80-120^{\circ}$, whereas the static culture had a stochastic orientation of $\sim 10 \%$ fibers found in each bin. $n=12 ; * * * * p<0.0001$.

Lastly, qualitative phalloidin staining of actin fibers and ICC against tenomodulin and DAPI in $\mathrm{iMSC}^{\mathrm{SCX}+}$ was performed. Following $7 \mathrm{~d}$ of uniaxial stretching, tenomodulin staining appeared to be brighter and was concentrated around nuclei. In contrast, the static culture tenomodulin staining displayed a less bright and much more diffuse pattern (Fig. 5). 

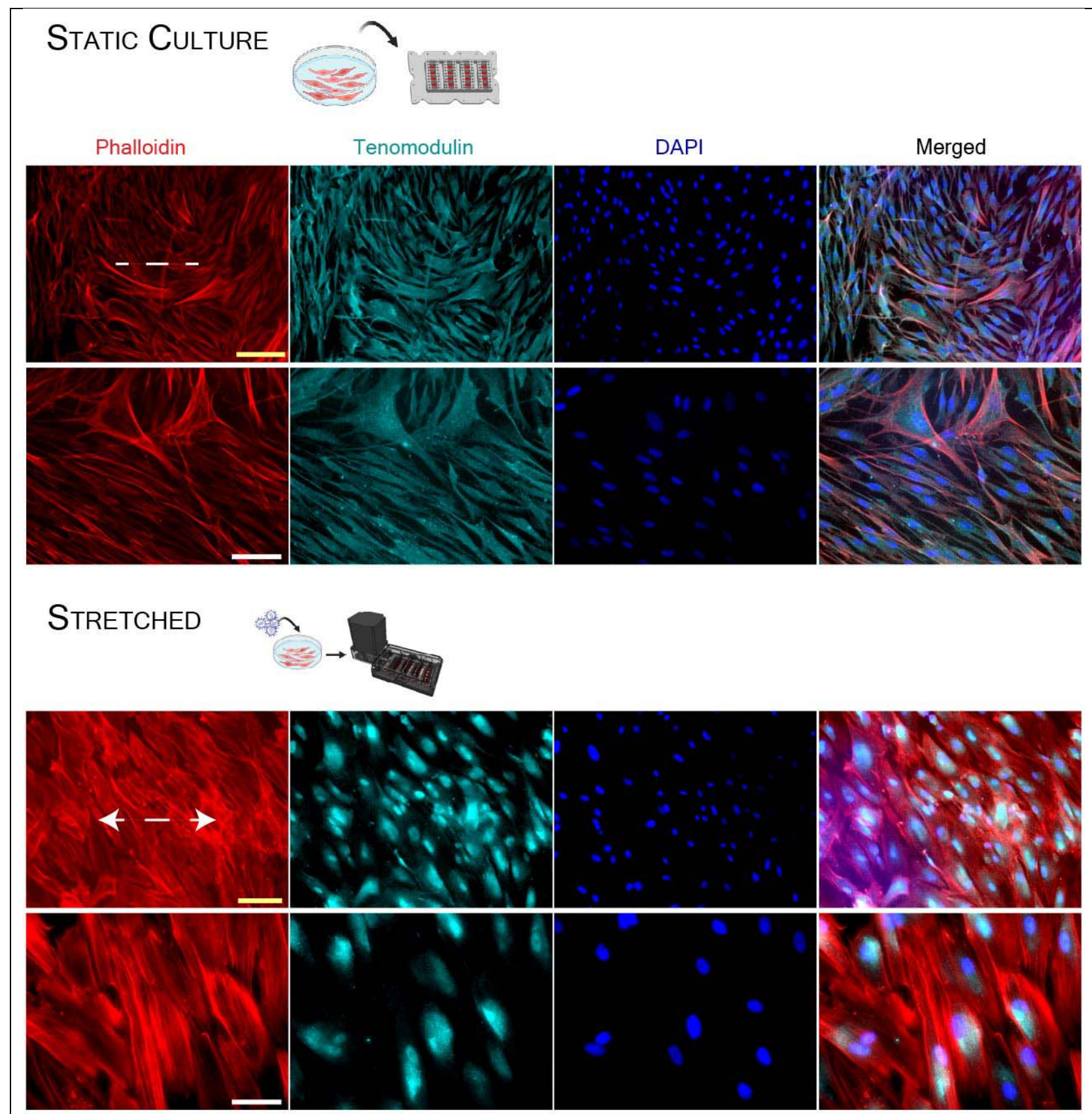

\section{Fig. 5. Confirmation of tenogenic differentiation on 2D bioreactor using}

immunocytochemistry. Cytoskeleton visualization using phalloidin staining of actin filaments (red), nucleic staining with DAPI (blue) and immunocytochemical staining for tenomodulin (cyan) was performed in the static culture ("static") and cyclic stretch ("stretched") conditions. 
Cells were seeded into silicone deformable plates with the same density. In the stretched condition, they were stretched uniaxially for 7 days in a $2 D$ bioreactor, while in the static they were placed in the incubator for the same timeframe. Channels are shown individually, and the merged images are shown on the right. Dotted line displays the long axis of the silicone plate which was the axis of the longitudinal stretch that was applied with this bioreactor system. Two different magnifications are shown for the two

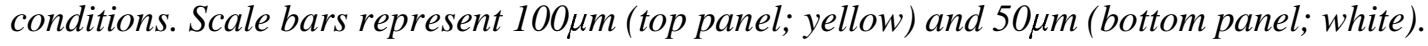

\section{Discussion}

In the present study we investigated the effect of combined stable Scx overexpression and biomechanical stimulation to induce tenogenic differentiation of BM-MSCs and iMSCs. We demonstrated the following: (a) lentiviral vector-mediated stable overexpression induced tenogenic differentiation in vitro (Fig. 1); (b) when altering substrate stiffness, cell culture on softer substrates did not promote tenogenic differentiation of BM-MSCs or iMSCs compared to standard tissue culture polystyrene with and without Scx overexpression (Fig. 2); (c) uniaxial cyclic stretching was able to induce upregulation of tenogenic markers in both iMSCs and $\mathrm{iMSC}^{\mathrm{SCX}+}$ at day 7, with significantly higher levels of expression in the $\mathrm{iMSC}^{\mathrm{SCX}+}$ group (Fig. 3); (d) uniaxial stretching resulted in changes in cell density, nuclear orientation and cytoskeleton alignment in $\mathrm{iMSC}^{\mathrm{SCX}+}$ cells (Fig. 4); and (e) Scx overexpression in iMSCs combined with stretching resulted in increased matrix and Tenomodulin deposition, indicating complementing effects of biological and mechanical cues to induce tenogenic differentiation in $\mathrm{iMSC}^{\mathrm{SCX}+}$ compared to iMSCs (Fig. 5). To our knowledge, this is the first study to demonstrate tenogenic differentiation processes in iPSC-derived $\mathrm{MSCs}^{\mathrm{SCX}+}$ under 2D cyclic tension. 
Scx stable overexpression resulted in a significant upregulation of earlier and later tendon markers in vitro, especially in iMSC ${ }^{\mathrm{SCX}+}$. These findings are in line with prior literature reporting that Scx is an important transcription factor that regulates syndetome specification and tendon

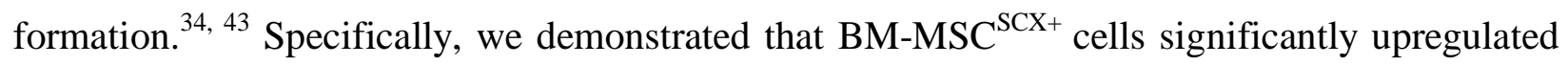
Bgn, Thbs4, and Dcn after 2 weeks of culture compared to their own baseline. Compared to naïve BM-MSCs, Thbs4 was increased and Tnmd decreased in BM-MSC ${ }^{\mathrm{SCX}+}$ cells (Fig. 1D). In contrast to our study, Alberton et al. demonstrated Scx transduction of the SCP-1 hTERT immortalized BM-MSC cell line to induce Tnmd expression in a static in vitro culture system. ${ }^{21}$ These differences may be a result of the cell source, since they used an immortalized cell line while our study tested primary low passage BM-MSCs. ${ }^{21}$

In the current study, iMSC ${ }^{\mathrm{SCX}+}$ showed a significant upregulation in a larger number of tenogenic markers, including Tnmd as well as Mkx, Tnc, Bgn, and Thbs4 compared to baseline and the other cell types that were assessed. The stronger response of the iMSCs to Scx overexpression might be due to differences in the cell origin: while BM-MSCs are obtained from different donors of different ages displaying phenotypic variability that are known to potentially affect their in vitro performance, iMSCs are considered a more homogeneous population of cells, since they are generated and expanded from a single origin. ${ }^{44}$ Moreover, iMSCs are derived from embryonic-like iPSCs and were reported to have a rejuvenation gene signature. ${ }^{45}$ In addition, iMSCs were shown to have superior survival and engraftment after transplantation, ${ }^{46}$ higher proliferative capacity, and lower senescence rate, ${ }^{47}$ and higher immunomodulation ability ${ }^{47,48}$ compared to BM-MSCs. 
The effect of substrate stiffness on the differentiation of multiple adult stem cells has been studied extensively. ${ }^{49-51}$ Mechanovariant substrates and selected ligands have been used in the past as a strategy to achieve directed differentiation of BM-MSCs towards tendon.$^{52,53}$ In the present study, use of mechanovariant substrates (stiffnesses of E 2kPa, 32kPa and GPa) did not promote expression of tenogenic differentiation markers compared to culture on standard TCP in BM-MSC and iMSC with and without Scx overexpression after 2 weeks of static culture (Fig. 2B). These results are in contrast with a previous study using BM-MSCs cultured at E $\sim 40 \mathrm{kPa}$ for 2 weeks, showing an upregulation of Scx, Tnmd, and Tnc. ${ }^{52}$ Differences between the results may be due to differences in donor or method of cell expansion prior to the experiments. ${ }^{54}$ For example, Li et al. found that stiff priming of rat BM-MSCs resulted in mechanical memory through a micro-RNA-21 control mechanism that lasted for more than 2 weeks. ${ }^{55}$

Tenocytes and tendon stem/progenitor cells dedifferentiate when cultured statically in regular tissue culture plates. ${ }^{7}$ Tnmd was immediately downregulated with in vitro culture of pig tenocytes, as has been reported. ${ }^{7}$ Interestingly, Alpl expression was close to baseline for the softer substrates, but significantly upregulated in TCP, which may indicate osteogenic differentiation of cultured tenocytes over time on more rigid substrates. ${ }^{56}$

In the present study, we stimulated iMSCs with $4 \%$ uniaxial strain and $0.5 \mathrm{~Hz}$ for $2 \mathrm{~h} /$ day in a $2 \mathrm{D}$ bioreactor using deformable silicone plates, which is in line with other studies showing that physiologically relevant loads range from $4 \%-6 \%$ strain at the cellular level of tendon. ${ }^{29}$ The final seeding density was chosen to avoid monolayer overgrowth on the silicone plate, which has been described to contribute to static tension. ${ }^{57}$

After 7 days of cyclic mechanical stimulation, a significant upregulation of Scx, Tnmd, Bgn, Tnc, Col1, Col3, Tppp3 as well as Pdgfra in stretched iMSCs compared to the static group was 
detected (Fig. 3A). Using the same bioreactor system (CellScale MCFX), but applying higher total strain amplitude and duration $(10 \%$ at $1 \mathrm{~Hz}$, for $12 \mathrm{~h} /$ day), Gaspar et al. were not able to detect changes in gene expression of Scx, Tnmd, and Colla1 and collagen deposition in BMMSCs and human tenocytes. ${ }^{57}$ However, they reported significant upregulation of Thbs4 after 3 days. This could reflect the higher strains that were applied in that study, since BM-MSCs aligned parallel to the load which is in contrast with a big body of research showing perpendicular cell alignment with physiological strains. ${ }^{58}$ In our study stretched iMSC ${ }^{\mathrm{SCX}+}$ significantly upregulated Thbs4, Sox9, and Pdgfra, but not Tnmd at day 7 compared to iMSCs (Fig. 3B). Comparable to our study, Chen et al. demonstrated an induction of Col1a1, Col1a2, Col14, and Tnmd and increased ECM deposition in lentiviral-mediated Scx-overexpressing hESC-MSCs that were assembled in multilayered cell sheets and cultured under uniaxial dynamic cyclic load (10\% strain, $1 \mathrm{~Hz}, 2 \mathrm{~h}$ /day) for up to 21 days. ${ }^{59}$ Implantation of these cells resulted in an improved tendon regeneration in a small-size tendon defect in a nude mouse model. $^{59}$

Assessment of new ECM deposition displayed significantly higher total collagen content in the iMSC $^{\mathrm{SCX}+}$ cells stretched for 7 days compared to iMSCs in both static and stretched conditions and to $\mathrm{iMSC}^{\mathrm{SCX}}$ in the static culture at the same timepoint (Fig. 3C). This indicates a potential synergistic effect of Scx overexpression and uniaxial loading resulting in not only tendon marker expression, but also more importantly secretion of ECM proteins to the media. BM-MSC ${ }^{\mathrm{SCX}+}$ in static culture and cell sheets of $\mathrm{Scx}^{+}$human ESCs-derived cells under loading have been shown to result in increased collagen deposition. ${ }^{21,59}$

In the statically cultured cells, a significantly higher cell density based on DAPI staining (Fig. 4A) was detected on day 7 , indicating that there might have been increased cell proliferation, 
since the same initial cell densities were used (Fig. 4B). This suggests that in the stretched $\mathrm{iMSC}^{\mathrm{SCX}+}$ mechanical stimulation resulted in reduced self-renewal compared to iMSCs, which is a characteristic of a more differentiated phenotype. ${ }^{21,60}$ Nucleic aspect ratios (width verus length) of stretched versus static cells was unaltered (Fig. 4C). However, the distribution of nuclear and cytoskeleton orientation was very distinct between the two conditions, suggesting that the cells re-oriented as a response to the stretch stimulus (Fig. 4D-E). While the statically cultured cells displayed stochastic nuclear arrangement in all directions, in the stretched cells the predominant orientation of nuclei was perpendicular to the applied load. Consistent with our findings, previous reports have shown cell orientation perpendicular to the stretching direction when physiological strains $(\leq \sim 7 \%)$ were applied. ${ }^{58,61}$

Actin filament staining and ICC for Tnmd in iMSC ${ }^{\mathrm{SCX}+}$ static and stretched cells showed diffuse localization of Tnmd in the entire cytoplasm (Fig. 5). In contrast, Tnmd seemed brighter and possibly more concentrated around the nuclei in the stretched group. Intriguingly, Tnmd gene expression was unchanged at days 3 and 7 with cyclic stretching in iMSC ${ }^{\mathrm{SCX}+}$ cells. Tnmd is directly regulated by Scx, ${ }^{62}$ and previous studies using Scx overexpression in BM-MSCs have reported Tnmd upregulation. ${ }^{21}$ However, recent findings using equine ESCs and fetal tenocytes to overexpress Scx have shown Tnmd gene downregulation, suggesting that mRNA and protein levels are differentially regulated and therefore need to be assessed in tandem. ${ }^{63}$

This study is not without limitations. It could be postulated that even though our transduction efficiency was close to $100 \%$, there may have been cells that were not transduced with Scx. Thus, the proportion of $\mathrm{Scx}^{+}$cells may decrease with expansion and some of the transduced cells might have lost Scx expression due to gene silencing. ${ }^{64}$ Cell sorting could potentially be used to obtain a purer iMSC ${ }^{\mathrm{SCX}+}$ population. Although, cell sorting was not necessary in this study, since 
we were able to achieve iMSC ${ }^{\mathrm{SCX}+}$ tenogenic differentiation, it may still be needed for future in vivo applications. Further, it has been postulated that the nominal strain applied in a 2D stretching system may be higher compared to the actual strain experienced by the cells. ${ }^{65}$ The latter may vary depending on bioreactor system, cell density, cell type (size, morphology, etc.), which hampers comparison and standardization between different studies. ${ }^{29,} 57$ To this end, we performed a series of pilot studies to optimize the stretch protocol and used complementary morphometric analyses to ensure we were stretching the cells within their physiological range. Lastly, the lack of adequate specific markers to assess temporal tenocyte gene expression changes has resulted in difficulty to characterize differentiation efforts and to discriminate between the effects of biological cues versus biomechanical ones that are crucial for tendon tissues. $^{25}$ Therefore, we need additional functional assessments to understand changes following differentiation efforts and the effects of mechanical stimulation on the maturation of the cellular phenotype.

\section{Conclusions}

An appropriate and potent cell therapeutic candidate for tendon regeneration is needed to address today's challenges in tendon and ligament repair. Our data provide evidence that iMSCs, genetically engineered to express Scx and stimulated by cyclic mechanical stretch to drive the cells into iTenocytes, may be a potential candidate for tendon cell therapy applications. Tenogenesis of $\mathrm{iMSC}^{\mathrm{SCX}+}$ was demonstrated by upregulation of early and late tendon markers, increased collagen deposition, and morphometric and cytoskeleton-related changes. Overall, iMSCs performed better than BM-MSCs, which might be due to the fact that iMSCs are developmentally more plastic. 
In future research, the functional impact of iTenocytes on tendon and ligament repair should be evaluated in an animal tendon injury model, providing an environment of physiological locomotion.

\section{Acknowledgments}

This study was supported by the NIH/NIAMS K01AR071512 to DS. The two lentivirus packaging plasmids were a gift by the Simon Knott laboratory (Department of Biomedical Sciences, Cedars-Sinai). The authors would like to thank Ms. Catherine Bresee (Cedars-Sinai Biostatistics Core Manager) for consultation on statistical analyses performed to compare the frequency distributions generated in the morphometric analyses.

\section{Disclosure of Potential Conflicts of Interest}

The authors have no conflict of interest. 


\section{References}

1. Nourissat G, Berenbaum F and Duprez D. Tendon injury: from biology to tendon repair. Nature Reviews Rheumatology 2015; 11: 223.

2. Chen J, Xu J, Wang A, et al. Scaffolds for tendon and ligament repair: review of the efficacy of commercial products. Expert review of medical devices 2009; 6: 61-73.

3. Baldino L, Cardea S, Maffulli N, et al. Regeneration techniques for bone-to-tendon and muscle-to-tendon interfaces reconstruction. British medical bulletin 2016; 117: 25-37.

4. Friel NA and Chu CR. The role of ACL injury in the development of posttraumatic knee osteoarthritis. Clinics in sports medicine 2013; 32: 1-12.

5. Ho JO, Sawadkar P and Mudera V. A review on the use of cell therapy in the treatment of tendon disease and injuries. Journal of Tissue Engineering 2014; 5: 2041731414549678.

6. Harvey T, Flamenco S and Fan C-M. A Tppp3+ Pdgfra+ tendon stem cell population contributes to regeneration and reveals a shared role for PDGF signalling in regeneration and fibrosis. Nature cell biology 2019; 21: 1490-1503.

7. Jo CH, Lim H-J and Yoon KS. Characterization of Tendon-Specific Markers in Various Human Tissues, Tenocytes and Mesenchymal Stem Cells. Tissue engineering and regenerative medicine 2019; 16: 151-159.

8. Lim WL, Liau LL, $\mathrm{Ng} \mathrm{MH}$, et al. Current progress in tendon and ligament tissue engineering. Tissue engineering and regenerative medicine 2019; 16: 549-571.

9. Gaspar D, Spanoudes K, Holladay C, et al. Progress in cell-based therapies for tendon repair. Advanced Drug Delivery Reviews 2015; 84: 240-256.

10. Abbah SA, Spanoudes K, O'Brien T, et al. Assessment of stem cell carriers for tendon tissue engineering in pre-clinical models. Stem cell research \& therapy 2014; 5: 38.

11. Gonçalves AI, Costa-Almeida R, Gershovich P, et al. Cell-based approaches for tendon regeneration. Tendon Regeneration. Elsevier, 2015, pp.187-203.

12. Dai L, Hu X, Zhang X, et al. Different tenogenic differentiation capacities of different mesenchymal stem cells in the presence of BMP-12. Journal of translational medicine $2015 ; 13$ : $1-14$.

13. Mehler VJ, Burns CJ, Stauss H, et al. Human iPSC-derived neural crest stem cells exhibit low immunogenicity. Molecular Therapy-Methods \& Clinical Development 2020; 16: 161-171.

14. Mehler VJ, Burns C and Moore ML. Concise review: exploring immunomodulatory features of mesenchymal stromal cells in humanized mouse models. Stem Cells 2019; 37: 298305.

15. Sheyn D, Ben-David S, Shapiro G, et al. Human induced pluripotent stem cells differentiate into functional mesenchymal stem cells and repair bone defects. Stem cells translational medicine 2016; 5: 1447-1460.

16. Czaplewski SK, Tsai T-L, Duenwald-Kuehl SE, et al. Tenogenic differentiation of human induced pluripotent stem cell-derived mesenchymal stem cells dictated by properties of braided submicron fibrous scaffolds. Biomaterials 2014; 35: 6907-6917.

17. Zhang C, Yuan H, Liu H, et al. Well-aligned chitosan-based ultrafine fibers committed teno-lineage differentiation of human induced pluripotent stem cells for Achilles tendon regeneration. Biomaterials 2015; 53: 716-730. 
18. $\mathrm{Xu} \mathrm{W}$, Wang Y, Liu E, et al. Human iPSC-derived neural crest stem cells promote tendon repair in a rat patellar tendon window defect model. Tissue Engineering Part A 2013; 19: 24392451.

19. Shukunami C, Takimoto A, Nishizaki Y, et al. Scleraxis is a transcriptional activator that regulates the expression of Tenomodulin, a marker of mature tenocytes and ligamentocytes. Scientific reports 2018; 8: 1-17.

20. Huang $\mathrm{AH}, \mathrm{Lu} \mathrm{HH}$ and Schweitzer R. Molecular regulation of tendon cell fate during development. Journal of Orthopaedic Research 2015; 33: 800-812.

21. Alberton P, Popov C, Prägert M, et al. Conversion of human bone marrow-derived mesenchymal stem cells into tendon progenitor cells by ectopic expression of scleraxis. Stem cells and development 2012; 21:846-858.

22. Chen D-Y, Crest J, Streichan SJ, et al. Extracellular matrix stiffness cues junctional remodeling for 3D tissue elongation. Nature communications 2019; 10: 1-15.

23. Riggin CN, Morris TR and Soslowsky LJ. Tendinopathy II: etiology, pathology, and healing of tendon injury and disease. Tendon Regeneration. Elsevier, 2015, pp.149-183.

24. Hockaday LA, Saeger MD, Karanja FW, et al. Mechanobiology of Embryonic and Adult Tendons. Tendon Regeneration. Elsevier, 2015, pp.77-110.

25. Glass ZA, Schiele NR and Kuo CK. Informing tendon tissue engineering with embryonic development. Journal of biomechanics 2014; 47: 1964-1968.

26. Ding S, Kingshott $\mathrm{P}$, Thissen $\mathrm{H}$, et al. Modulation of human mesenchymal and pluripotent stem cell behavior using biophysical and biochemical cues: A review. Biotechnology and bioengineering 2017; 114: 260-280.

27. Tatullo M, Marrelli M, Falisi G, et al. Mechanical influence of tissue culture plates and extracellular matrix on mesenchymal stem cell behavior: A topical review. SAGE Publications Sage UK: London, England, 2016.

28. Ryan $\mathrm{CN}$ and Zeugolis DI. Engineering the Tenogenic Niche In Vitro with Microenvironmental Tools. Advanced Therapeutics 2020; 3: 1900072.

29. Wang T, Chen P, Zheng M, et al. In vitro loading models for tendon mechanobiology. Journal of Orthopaedic Research® 2018; 36: 566-575.

30. Sheyn D, Ben-David S, Tawackoli W, et al. Human iPSCs can be differentiated into notochordal cells that reduce intervertebral disc degeneration in a porcine model. Theranostics 2019; 9: 7506-7524. 2019/11/07. DOI: 10.7150/thno.34898.

31. Chahla J, Papalamprou A, Chan V, et al. Assessing the Resident Progenitor Cell Population and the Vascularity of the Adult Human Meniscus. Arthroscopy : the journal of arthroscopic \& related surgery : official publication of the Arthroscopy Association of North America and the International Arthroscopy Association 2021; 37: 252-265. 2020/09/27. DOI: 10.1016/j.arthro.2020.09.021.

32. Glaeser JD, Behrens P, Stefanovic T, et al. Neural crest $\square$ derived mesenchymal progenitor cells enhance cranial allograft integration. Stem cells translational medicine 2021; 10: 797-809.

33. Stewart SA, Dykxhoorn DM, Palliser D, et al. Lentivirus-delivered stable gene silencing by RNAi in primary cells. Rna 2003; 9: 493-501.

34. Pryce BA, Brent AE, Murchison ND, et al. Generation of transgenic tendon reporters, ScxGFP and ScxAP, using regulatory elements of the scleraxis gene. Developmental dynamics: an official publication of the American Association of Anatomists 2007; 236: 1677-1682. 
35. Falcon ND, Riley GP and Saeed A. Induction of tendon-specific markers in adiposederived stem cells in serum-free culture conditions. Tissue Engineering Part C: Methods 2019; 25: 389-400.

36. Perucca Orfei C, Viganò M, Pearson JR, et al. In vitro induction of tendon-specific markers in tendon cells, adipose-and bone marrow-derived stem cells is dependent on TGF $\beta 3$, BMP-12 and ascorbic acid stimulation. International journal of molecular sciences 2019; 20: 149.

37. Garvin J, Qi J, Maloney M, et al. Novel system for engineering bioartificial tendons and application of mechanical load. Tissue engineering 2003; 9: 967-979.

38. Brown JP, Finley VG and Kuo CK. Embryonic mechanical and soluble cues regulate tendon progenitor cell gene expression as a function of developmental stage and anatomical origin. Journal of biomechanics 2014; 47: 214-222.

39. Gertych A, Ma Z, Tajbakhsh J, et al. Rapid 3-D delineation of cell nuclei for high-content screening platforms. Computers in biology and medicine 2016; 69: 328-338.

40. Russo V, Mauro A, Martelli A, et al. Cellular and molecular maturation in fetal and adult ovine calcaneal tendons. Journal of anatomy 2015; 226: 126-142.

41. Jelinsky SA, Archambault J, Li L, et al. Tendon $\square$ selective genes identified from rat and human musculoskeletal tissues. Journal of Orthopaedic Research 2010; 28: 289-297.

42. Schmittgen TD and Livak KJ. Analyzing real-time PCR data by the comparative C T method. Nature protocols 2008; 3: 1101.

43. Brent AE, Schweitzer R and Tabin CJ. A somitic compartment of tendon progenitors. Cell 2003; 113: 235-248.

44. Chow L, Johnson V, Regan D, et al. Safety and immune regulatory properties of canine induced pluripotent stem cell-derived mesenchymal stem cells. Stem cell research 2017; 25: 221 232.

45. Spitzhorn LS, Megges M, Wruck W, et al. Human iPSC-derived MSCs (iMSCs) from aged individuals acquire a rejuvenation signature. Stem cell research \& therapy 2019; 10: 100. 20190318. DOI: 10.1186/s13287-019-1209-X.

46. Kang R, Zhou Y, Tan S, et al. Mesenchymal stem cells derived from human induced pluripotent stem cells retain adequate osteogenicity and chondrogenicity but less adipogenicity. Stem cell research \& therapy 2015; 6: 144. 20150818. DOI: 10.1186/s13287-015-0137-7.

47. Gao WX, Sun YQ, Shi J, et al. Effects of mesenchymal stem cells from human induced pluripotent stem cells on differentiation, maturation, and function of dendritic cells. Stem cell research \& therapy 2017; 8: 48. 20170302. DOI: 10.1186/s13287-017-0499-0.

48. Wang LT, Jiang SS, Ting $\mathrm{CH}$, et al. Differentiation of Mesenchymal Stem Cells from Human Induced Pluripotent Stem Cells Results in Downregulation of c-Myc and DNA Replication Pathways with Immunomodulation Toward CD4 and CD8 Cells. Stem Cells 2018; 36: 903-914. 20180212. DOI: 10.1002/stem.2795.

49. Skardal A, Mack D, Atala A, et al. Substrate elasticity controls cell proliferation, surface marker expression and motile phenotype in amniotic fluid-derived stem cells. Journal of the mechanical behavior of biomedical materials 2013; 17: 307-316.

50. Crowder SW, Leonardo V, Whittaker T, et al. Material cues as potent regulators of epigenetics and stem cell function. Cell stem cell 2016; 18: 39-52.

51. Engler AJ, Sen S, Sweeney HL, et al. Matrix elasticity directs stem cell lineage specification. Cell 2006; 126: 677-689. 
52. Sharma RI and Snedeker JG. Biochemical and biomechanical gradients for directed bone marrow stromal cell differentiation toward tendon and bone. Biomaterials 2010; 31: 7695-7704.

53. Sharma RI and Snedeker JG. Paracrine interactions between mesenchymal stem cells affect substrate driven differentiation toward tendon and bone phenotypes. PloS one 2012; 7: e31504.

54. Yin JQ, Zhu J and Ankrum JA. Manufacturing of primed mesenchymal stromal cells for therapy. Nature biomedical engineering 2019; 3: 90-104.

55. Li CX, Talele NP, Boo S, et al. MicroRNA-21 preserves the fibrotic mechanical memory of mesenchymal stem cells. Nature materials 2017; 16: 379-389.

56. Sun M, Chi G, Xu J, et al. Extracellular matrix stiffness controls osteogenic differentiation of mesenchymal stem cells mediated by integrin $\alpha 5$. Stem cell research \& therapy 2018; 9: 1-13.

57. Gaspar D, Ryan CN and Zeugolis DI. Multifactorial bottom $\square$ up bioengineering approaches for the development of living tissue substitutes. The FASEB Journal 2019; 33: 57415754.

58. Chagnon-Lessard S, Jean-Ruel H, Godin M, et al. Cellular orientation is guided by strain gradients. Integrative Biology 2017; 9: 607-618.

59. Chen X, Yin Z, Chen J-1, et al. Force and scleraxis synergistically promote the commitment of human ES cells derived MSCs to tenocytes. Scientific reports 2012; 2: 1-9.

60. Chacón-Martínez CA, Koester J and Wickström SA. Signaling in the stem cell niche: regulating cell fate, function and plasticity. Development 2018; 145: dev165399.

61. Morita Y, Watanabe S, Ju Y, et al. Determination of optimal cyclic uniaxial stretches for stem cell-to-tenocyte differentiation under a wide range of mechanical stretch conditions by evaluating gene expression and protein synthesis levels. Acta of bioengineering and biomechanics 2013; 15.

62. Liu H, Xu J, Lan Y, et al. The Scleraxis transcription factor directly regulates multiple distinct molecular and cellular processes during early tendon cell differentiation. Frontiers in cell and developmental biology 2021; 9: 1372.

63. Bavin EP, Atkinson F, Barsby T, et al. Scleraxis is essential for tendon differentiation by equine embryonic stem cells and in equine fetal tenocytes. Stem cells and development 2017; 26: 441-450.

64. Vranckx LS, Demeulemeester J, Debyser Z, et al. Towards a safer, more randomized lentiviral vector integration profile exploring artificial LEDGF chimeras. PloS one 2016; 11: e0164167.

65. Wall ME, Weinhold PS, Siu T, et al. Comparison of cellular strain with applied substrate strain in vitro. Journal of biomechanics 2007; 40: 173-181. 